\title{
Artificial Neural Networks Study on Prediction of Dielectric Permittivity of Basalt/PANI Composites
}

\author{
Onder Eyecioglu*๋, Mehmet Kilic**, Yasar Karabul **, Umit Alkan***, Orhan Icelli** \\ *Department of Mechatronics Engineering, Faculty of Engineering and Architecture, Istanbul Gelişim University, 34215, \\ Istanbul, Turkey. \\ **Department of Physics, Faculty of Science and Letters, Yildiz Technical University, 34220 Istanbul, Turkey. \\ ***Department of Computer Engineering, Faculty of Engineering and Architecture, Istanbul Gelişim University, 34215, \\ Istanbul, Turkey. \\ (oeyecioglu@gelisim.edu.tr, mekilic@yildiz.edu.tr, karabul@yildiz.edu.tr, ualkan@gelisim.edu.tr, oicelli@yildiz.edu.tr)
}

${ }^{\star}$ Corresponding Author: Onder Eyecioglu, Department of Mechatronics Engineering, Faculty of Engineering and Architecture,
Istanbul Gelisim University, 34215, Istanbul, Turkey, Tel: +90 $2124227020 / 288$, oeyecioglu@ gelisim.edu.tr

Received: 29.03.2016 Accepted: 30.04.2016

\begin{abstract}
In the present study, the dielectric permittivity change of basalt (two type basalt; CM-1, KYZ-13) reinforced PANI composites were studied to determine the effects of PANI additivities (10.0, 25.0, $50.0 \mathrm{wt} . \%)$ at several frequencies from 100 $\mathrm{Hz}$ to $17.5 \mathrm{MHz}$ by a dielectric spectroscopy method at the room temperature and artificial neural networks (ANNs) simulation. Also, the dielectric permittivity at $30.0 \mathrm{wt} . \%$ of PANI additivity was obtained by ANNs without experimental process. That process, a significant predictive instrument was produced which allows optimization of dielectric properties for numerous composites without substantial experimentation. It has been observed that PANI additivities decreased to dielectric constant of composites at low frequencies. Furthermore, the ANNs method have satisfactory accuracy for prediction of dielectric parameters.
\end{abstract}

Keywords: Artificial neural networks, dielectric permittivity, basalt, PANI, composite.

\section{Introduction}

There is growing interest in reinforcing polymer matrix composites with basalt reinforced polymers because of their moderate cost, high stiffness and strength, excellent corrosion and oxidation resistance, and heat resistance and thermal stability. Basalt is a very common volcanic rock, dark colored and comparatively rich in iron and magnesium, which is located at almost every country in the world. Basalt is used for a wide variety of purposes. It has been used in the rock industry to create industrial construction, highway engineering and building tiles for other purposes [1]. Polyaniline (PANI) is one of the most promising conductive polymers for technological applications due to its easy synthesis, high environmental stability, huge electrical conductivity, as well as a comparatively low cost [2, 3]. PANI is synthesized for specific applications like organic electronics [3], circuit component (such as varistors [4], super capacitors [3, 5]), electrochemical catalysis [5, 6], corrosion protection $[7,8]$ and sensors [9].
Artificial neural networks (ANNs) are becoming wellknown because of their achievement where complicated nonlinear relationships occur amongst data. ANNs are biologically influenced computer programs created to simulate the way in which the human brain processes knowledge. ANNs collect this information through detecting the relationships and patterns in learned via experience and data. The broad use of ANNs is a result of their ability and versatility to model nonlinear systems without earlier information of an empirical model. They do not require a specific formulation of the physical or mathematical relationships of the undertaking issue. These give ANNs an advantage over traditional fitting methods for numerous application. [10].

In this work, the dielectric permittivity values of basalt reinforced PANI were determined at several frequencies from $100 \mathrm{~Hz}$ to $17.5 \mathrm{MHz}$ by using experimental Impedance spectroscopy technique, and this data was used to develop an artificial neural networks model for next prediction of dielectric permittivity. The data samples were produced three different composite $(10.0,25.0,50.0$ wt.\%). Then, we 
calculated the dielectric permittivity for 30.0 wt.\% PANI additivity as a sample application of developed ANNs model.

\section{Materials and Method}

\subsection{Materials}

Basalt samples (two type basalt samples and coded CM1, KYZ-13) obtained from different regions of Van in Turkey. Chemical analyses of the basalt samples are taken by $\mathrm{X}$-ray fluorescence (XRF) instrument. Operating conditions of the XRF device of Philips PW-2400 were fixed at $50 \mathrm{~mA}$ and $60 \mathrm{kV}$. The results of chemical analysis of basalt samples are given in Table 1.

Table 1. Chemical composition of basalt samples [1].

\begin{tabular}{|c|c|c|}
\hline Sample & CM-1 & KYZ-13 \\
\hline $\mathrm{SiO}_{2}$ & 41.668 & 47.790 \\
\hline $\mathrm{TiO}_{2}$ & 2.0800 & 1.3950 \\
\hline $\mathrm{Al}_{2} \mathrm{O}_{3}$ & 13.106 & 16.918 \\
\hline $\mathrm{Fe}_{2} \mathrm{O}_{3}$ & 13.823 & 10.878 \\
\hline MnO & 0.1920 & 0.1630 \\
\hline MgO & 9.7540 & 7.6190 \\
\hline $\mathrm{CaO}$ & 10.602 & 11.357 \\
\hline $\mathrm{Na}_{2} \mathrm{O}$ & 5.2610 & 3.1370 \\
\hline $\mathrm{K}_{2} \mathrm{O}$ & 1.7370 & 0.5190 \\
\hline $\mathbf{P}_{2} \mathbf{O}_{5}$ & 1.7770 & 0.2240 \\
\hline
\end{tabular}

Polyaniline (emeraldine base, average Mw 5000) was purchased from Sigma-Aldrich. To investigate effects of PANI additive percentages $(10.0,25.0,50.0 \mathrm{wt} \%)$ on the dielectric properties of basalt mechanically modified by PANI. These composites were prepared by mixing of basalt samples with PANI and were molded by compression in a cold press at room temperature. Each one of these mixtures prepared as a pellet having $13 \pm 0.02 \mathrm{~mm}$ diameter, $0.500 \pm 0.050 \mathrm{~g}$ weight and 1.9-2.2 mm thickness.

\subsection{Dielectric Measurements}

The dielectric measurements were carried out with a twopoint probe arrangement. Dielectric measurements have been performed by using an HP 4194A Impedance Analyzer in the frequency range from $100 \mathrm{~Hz}-15 \mathrm{MHz}$ at room temperature with a high accuracy $(0.17 \%$ typ.) In this work, the overall errors the dielectric measurements are $2.5 \%$ and the RMS amplitude of the instruments is $\sim 500 \mathrm{mV}$.

\subsection{Artificial Neural Networks (ANNs)}

ANNs are a mathematical model that can be defined as an interconnected set of nodes their connections. Also neural network is a network of neurons. The neurons are the predefined computational units that are grouped in sets of layers. Basically, there are three types of layers, input, output and hidden layer as seen in Fig. 1. The input layer receives data from the outside and transfers them to sub layers. The data comes from input layer is processed in the hidden layer by performing interconnection of neurons and sending the results to the neuron in the output layer.

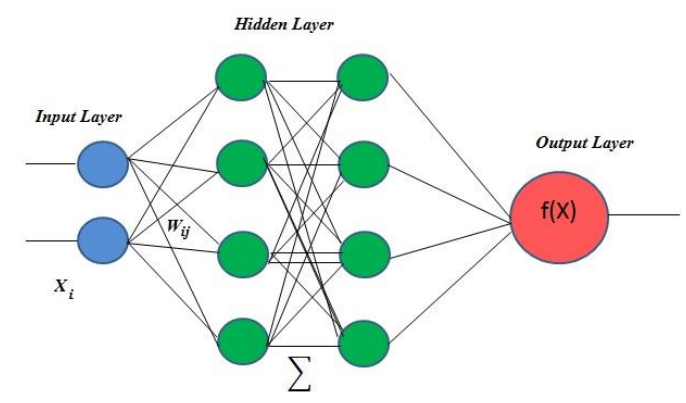

Fig. 1. The architecture of the ANNs algorithm.

The output layer submits the output data. The neurons perform weighted summation of the inputs that are applied to the networks calculated as [11];

$$
N e t_{i}=\sum_{j=1}^{N} X_{i j} \cdot W_{i j}+n_{i}
$$

Where $W_{i j}$ is the weight between the $i$ th neuron in the previous layer to the $j$ th neuron. $X_{i j}$ is the output of $i$ th neuron and $n_{i}$ is the bias value of previous layer.

The output of the $i$ th neuron is calculated using a sigmoidal activation function as follows.

$$
\text { out }_{i}=f\left(N e t_{i}\right)=\frac{1}{1+e^{\left(-a N e t_{i}\right)}}
$$

Here, $a$ is a constant used for controlling the slope of the semi-linear region [11].

There are two stages to process the network for an ANNs model. First of them is training and the other is testing. In the training stage, the network is training to predict an output based on the input. After that the training stage, the predicted output is compared with given output. This testing decides whether to stop or continue training of the network.

For the training of the network, a back propagation algorithm (BP) has been used. The Levenberg - Marquard (LM) algorithm $[12,13]$ is used to update the weights $\left(W_{i j}\right)$ for minimizing the error function that defined as absolute difference between predicted output value and given actual value. In the LM algorithm, the Hessian matrix can be approximated as;

$$
H=J^{T} J
$$

and this approximation is used for the iteration of the weights;

$$
x_{k+1}=x_{k}-\left[J^{T} J-\mu I\right]^{-1} J^{T} e
$$

Where $J$ is the Jacobian matrix, $e$ is a vector of network errors, $\mu$ is the scalar learning rate and $I$ is the identity matrix. When $\mu$ is large, this becomes gradient descent method. This method used as adaption function in this study. 
The prediction accuracy performance of the networks was calculated using coefficient of determination $(R)$ and relative error $(R E)$;

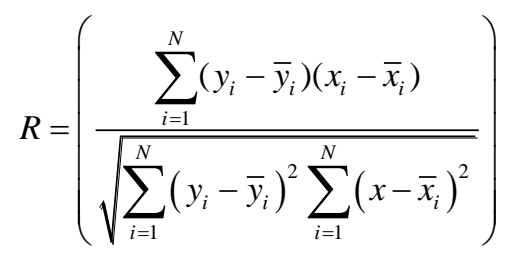

$\operatorname{RE}(\%)=\frac{\left|x_{i}-y_{i}\right|}{x_{i}} \times 100$

where $y_{i}$ and $x_{i}$ are predicted and actual values respectively.

\section{Results}

Dielectric spectroscopy is most reliable and powerful experimental technique which has been effectively used for the characterization of electrical properties of the polar materials [14]. The DS technique is based on analyzing the alternative current (a.c.) response of a materials to a sinusoidal perturbation, and subsequent calculation of dielectric parameters as a function of frequency and temperature [15].

The dielectric analysis gives the permittivity and conductivity of material as a complex permittivity $\varepsilon^{*}(\omega)$ parameter. Dielectric permittivity is a principal parameter that determines the coupling and distribution of electromagnetic energy during microwave and
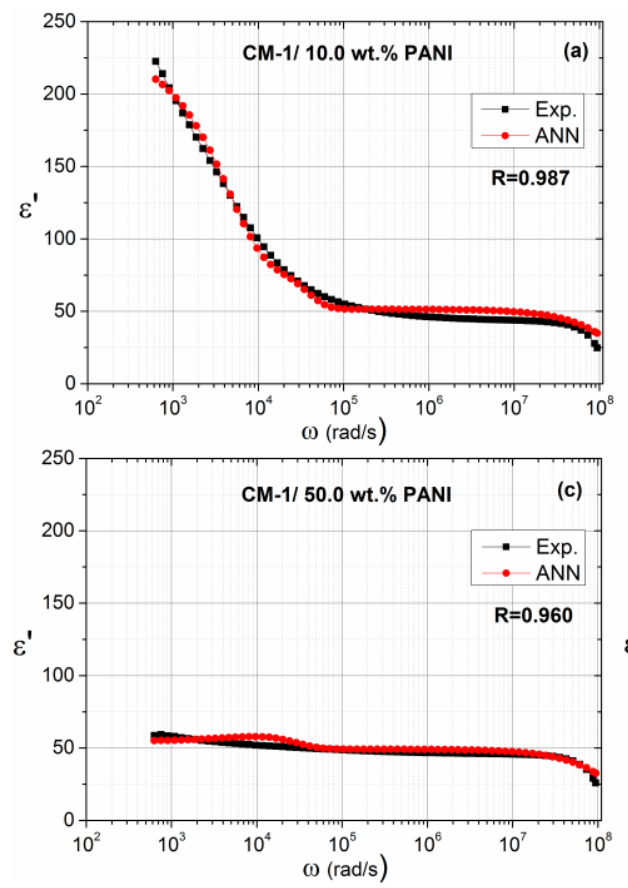

radiofrequency processing $[15,16]$. The frequency dependence complex dielectric permittivity $\varepsilon^{*}(\omega)$ is given by

$$
\varepsilon^{*}(\omega)=\varepsilon^{\prime}(\omega)+i \varepsilon^{\prime \prime}(\omega)
$$

Here, $\omega, \varepsilon^{\prime}(\omega)$ and $\varepsilon^{\prime \prime}(\omega)$ are the angular frequency, the real part of complex permittivity (or called dielectric constant) and the imaginary part of the complex dielectric permittivity (or called loss factor), respectively. In dielectric material $\varepsilon^{\prime}(\omega)$ represents the alignment of dipoles, which is the energy storage component and $\varepsilon^{\prime \prime}(\omega)$ represents the ionic conduction component [15].

In this study, experimental results of $\varepsilon^{\prime}(\omega)$ and $\varepsilon^{\prime \prime}(\omega)$ for samples have been shown in Fig. 2-5. The increase of the doping percentage of PANI decreases the dielectric constant for both composites in low-frequencies. Moreover, the $\varepsilon^{\prime}(\omega)$ and $\varepsilon^{\prime \prime}(\omega)$ have large values in the low-frequency region and decreases rapidly in $10.0 \mathrm{wt}$ \% PANI composites.

Furthermore, the ANNs model is developed to predict the dielectric parameters $\left(\varepsilon^{\prime}(\omega)\right.$ and $\left.\varepsilon^{\prime \prime}(\omega)\right)$ of these composites. The experimental measurements are used to obtain the 200 data samples of dielectric parameters $\left(\varepsilon^{\prime}(\omega)\right.$, $\varepsilon^{\prime \prime}(\omega)$ ) for various concentration (wt.\%) and frequency $(\omega)$. Concentration and frequency of 200 data samples are used as input and, dielectric parameters of corresponding them are used as output (target). $80 \%$ percent of the data are used as training data and the other $20 \%$ are the testing data. The training and testing data are selected us randomly.
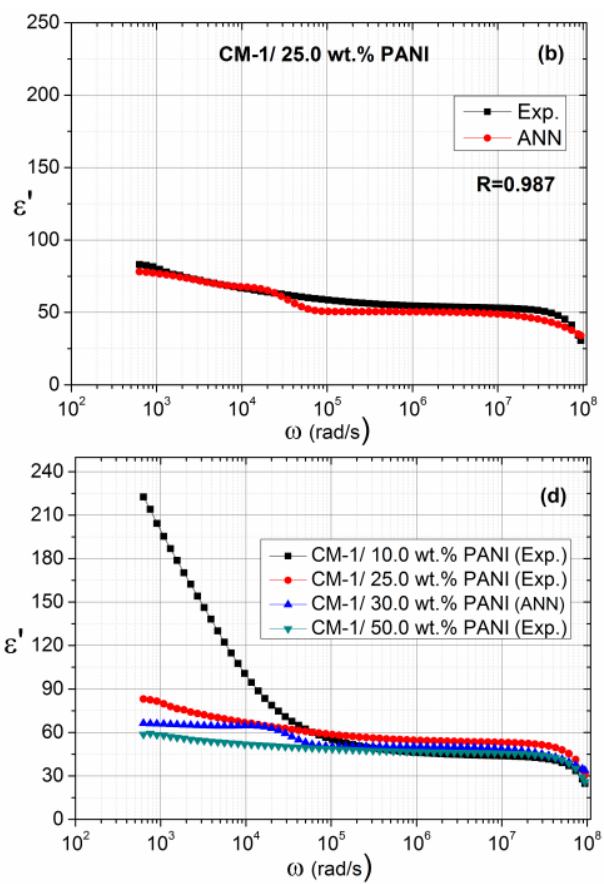

Fig. 2. Comparison of desired experimental results and predicted values of $\varepsilon^{\prime}(\omega)$ for samples of (a) CM-1/ $10.0 \mathrm{wt}$ \% PANI

(b) CM-1/ 25.0 wt.\% PANI (c) CM-1/ 50.0 wt.\% PANI composites and (d) $\varepsilon^{\prime}(\omega)$ prediction of CM-1/ 50.0 wt.\% PANI composite 

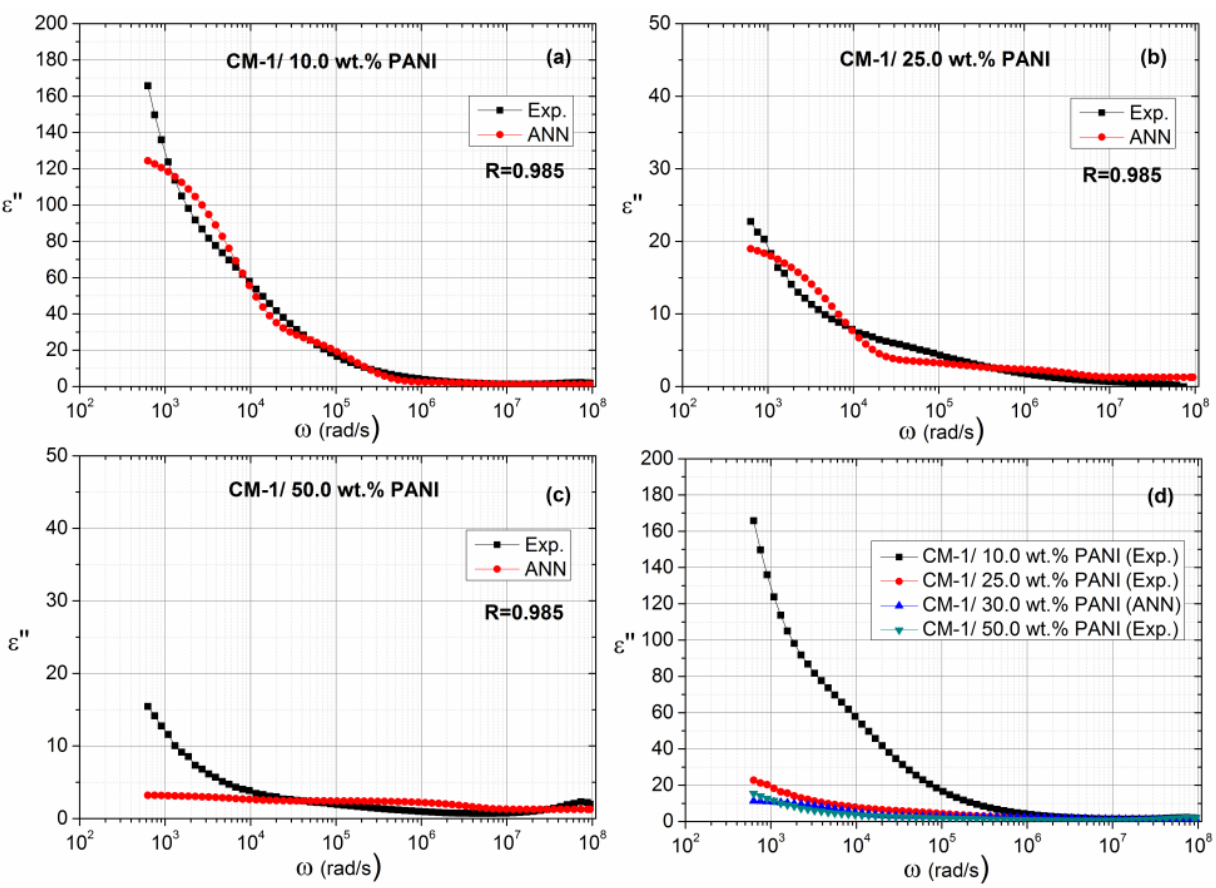

Fig. 3. Comparison of desired experimental results and predicted values of $\varepsilon^{\prime \prime}(\omega)$ for samples of (a) CM-1/ 10.0 wt.\% PANI

(b) CM-1/ 25.0 wt.\% PANI (c) CM-1/ 50.0 wt.\% PANI composites and (d) $\varepsilon^{\prime \prime}(\omega)$ prediction of CM-1/ 30.0 wt.\% PANI composite

The comparisons of experimental results and output values predicted by ANNs of CM-1 are illustrated in Fig. 23 . The figures show the variation of $\varepsilon^{\prime}(\omega)$ and $\varepsilon^{\prime \prime}(\omega)$ with the frequency for various concentration (10.0 wt.\%, 25.0 wt.\% and 50.0 wt.\%,). From these figures, it is seen that the high accuracy between experimental and simulated data.
Therefore, it can be concluded easily that the outputs efficiently modeled by the ANNs model.

Also, it can be concluded from Fig. 4 and Fig. 5, that the KYZ-13 values can be efficiently modeled by the ANNs model similar to the CM-1.
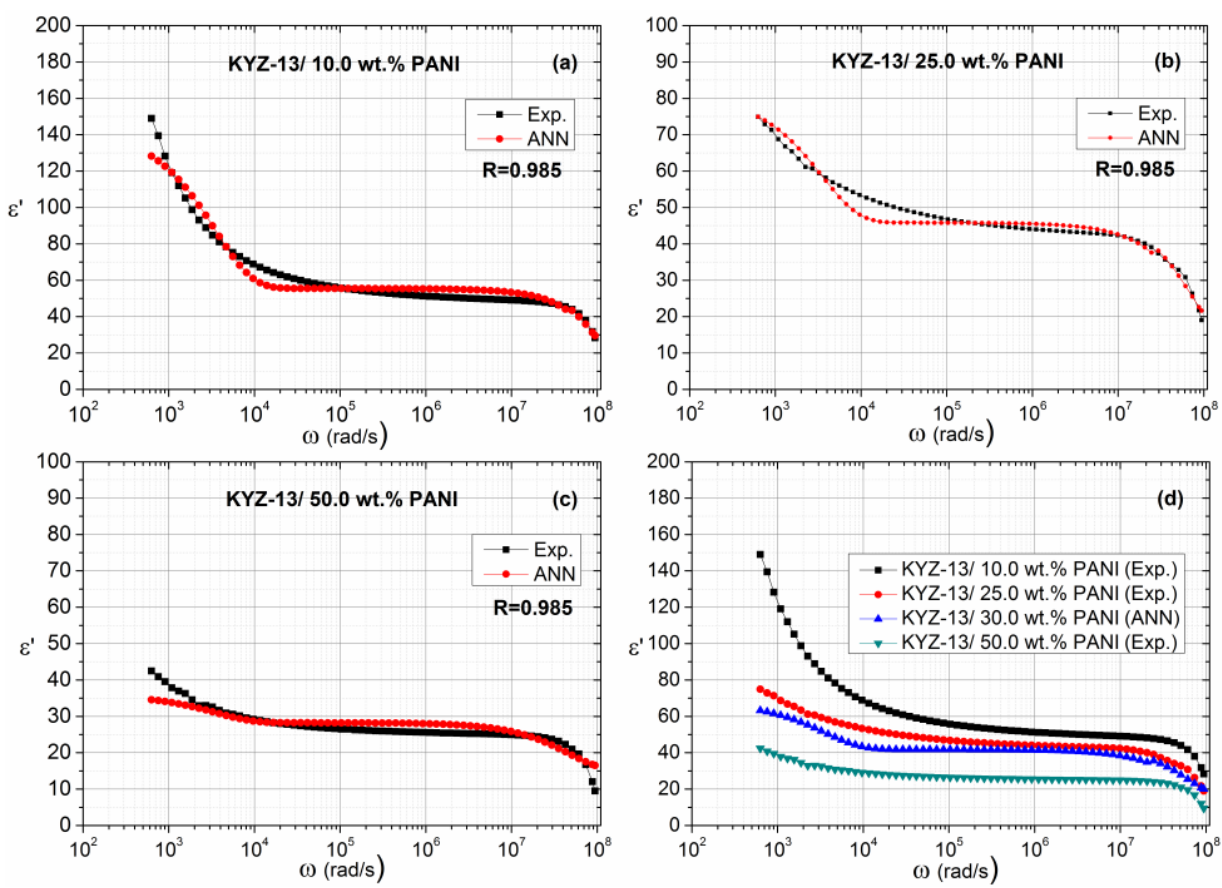

Fig. 4. Comparison of desired experimental results and predicted values of $\varepsilon^{\prime}(\omega)$ for samples of (a) KYZ-13/ 10.0 wt.\% PANI

(b) KYZ-13/ 25.0 wt.\% PANI (c) KYZ-13/ 50.0 wt.\% PANI composites and (d) $\varepsilon^{\prime}(\omega)$ prediction of KYZ-13/ 50.0 wt.\%

PANI composite 

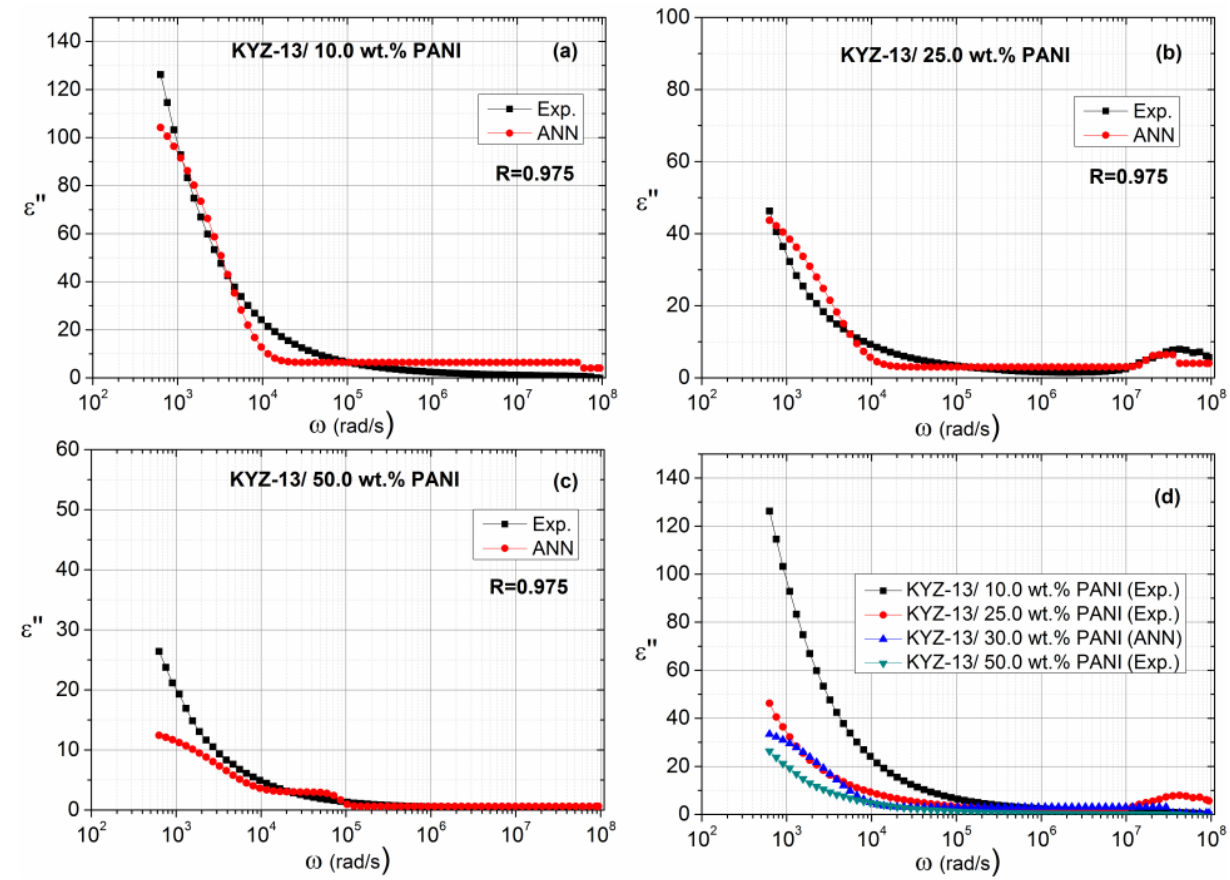

Fig. 5. Comparison of desired experimental results and predicted values of $\varepsilon^{\prime \prime}(\omega)$ for samples of (a) KYZ-13/ 10.0 wt.\% PANI

(b) KYZ-13/ 25.0 wt.\% PANI (c) KYZ-13/ 50.0 wt.\% PANI composites and (d) $\varepsilon^{\prime \prime}(\omega)$ prediction of KYZ-13/ 30.0 wt.\%

PANI composite

By using efficient training algorithm, the output values of $\varepsilon^{\prime}(\omega)$ and $\varepsilon^{\prime \prime}(\omega)$ has been predicted for CM-1/ 30.0 wt. \% PANI samples that were not measured in our experiments. The variation of predicted $\varepsilon^{\prime}(\omega)$ and $\varepsilon^{\prime \prime}(\omega)$ values are figured in Fig. 2-d, Fig. 3-d, Fig. 4-d and Fig. 5-d. It can be seen that suitable output values can be obtained from ANN model for $\varepsilon^{\prime}(\omega)$ and $\varepsilon^{\prime \prime}(\omega)$.

The performance of the model on training and testing stages in terms of $R$ is presented in Fig. 6 and Fig. 7.
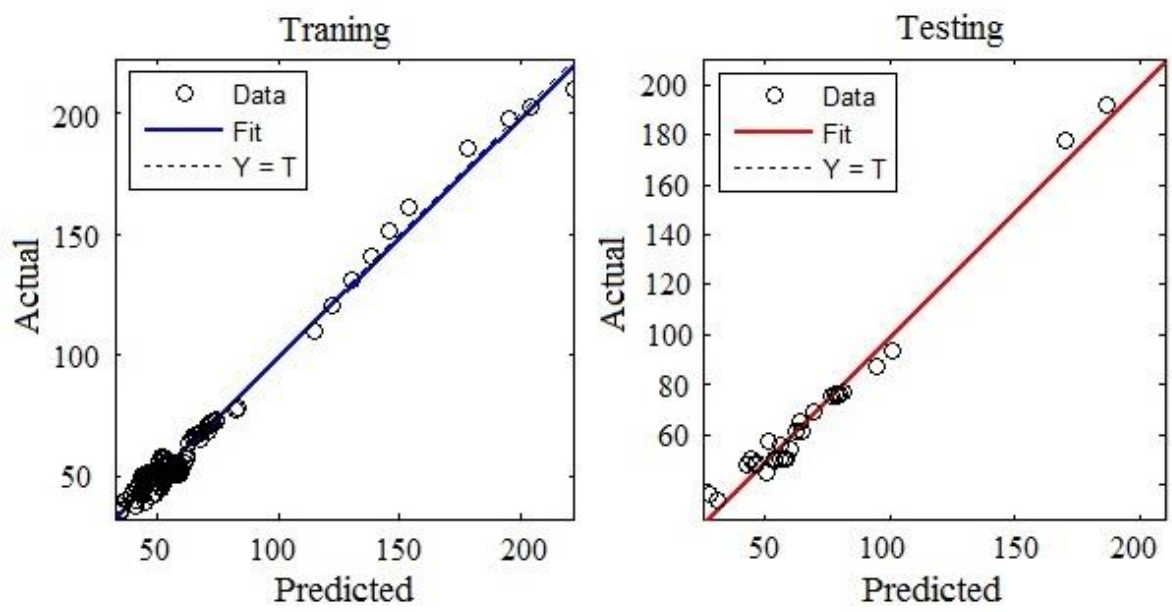

Fig. 6. Fit of ANNs model on (a) training data and (b) testing data of CM-1/PANI composites 

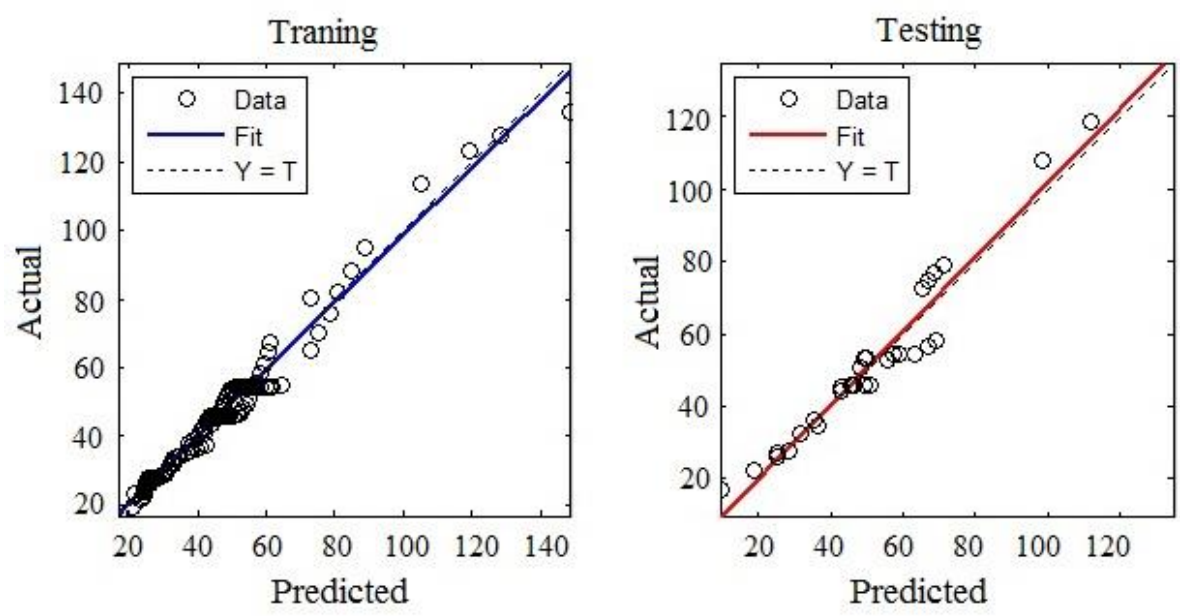

Fig. 7. Fit of ANNs model on (a) training data and (b) testing data of KYZ-13/PANI composites

The graphs show that the training algorithm worked well, and learned the non-linear relation between concentration, frequency and dielectric constant with high $R$ value for both CM-1 and KYZ-13 samples. The $R$ values of both training and testing are given in Table 2.

Table 2. Coefficient of determination $(R)$ parameters of ANN for CM-1 and KYZ-13 composites

\begin{tabular}{|c|c|c|}
\hline $\boldsymbol{R}$ & SM-1 & KYZ-13 \\
\hline $\boldsymbol{R}$ (Training) & 0.991 & 0.987 \\
\hline $\boldsymbol{R}$ (Testing) & 0.989 & 0.972 \\
\hline
\end{tabular}

\section{Conclusion}

This study represents the results of our investigation on the dielectric properties of two type Basalt/PANI composite using dielectric spectroscopy and an application of the ANNs method in the prediction of dielectric properties of basalt materials depend on additive percentage $(\%)$ and frequency $(\omega)$.

The results given in this paper prove that the ANN method has satisfactory accuracy for prediction of desired properties ( $\varepsilon^{\prime}(\omega)$ and $\varepsilon^{\prime \prime}(\omega)$ ). So the ANNs model can be used as an appropriate and practical method to compute dielectric properties of materials under various experimental conditions such as, concentration, frequency or temperature.

\section{Acknowledgements}

This work was supported by TUBİTAK under award number 2015-115F311. This study has also been supported by Yildiz Technical University Scientific Research Projects Coordination Department under Project number: 2015-0101-GEP03.

\section{References}

[1] Y. Karabul, L.A. Susam, O. İçelli and Ö. Eyecioğlu,
"Computation of EABF and EBF for basalt rock samples", Nucl. Instrum. Methods. Phys. Res. A, vol. 797, pp. 29-36, 2015.

[2] H.S. Nalwa, Handbook of Organic Conductive Molecules and Polymers, New York: Wiley, 1997.

[3] Z.A. Boeva and V.G. Sergeyev, "Polyaniline: Synthesis, Properties, and Application", Polymer Science Series C vol. 56, pp. 144-153, 2014.

[4] H. Bidadi, A. Olad, M. Parhizkar, S.M. Aref and M. Ghafouri, "Nonlinear Properties of ZnO-Polymer Composites Prepared by Solution-casting Method", Vacuum, vol. 87, pp. 50-54, 2013.

[5] H. Tang, Y. Ding, C. Zang, J. Gu, Q. Shen and J. Kan, "Effect of Temperature on Electrochemical Degradation of Polyaniline", Int. J. Electrochem. Sci., vol. 9, pp. 7252-7239, 2014.

[6] Z. Wen-Zhi , K. Xian-Wen, J. Shou-Feng, S. Jin-Gao, Y. Dong-Sheng and F. Bin, "Electrochemical characteristics and catalytic activity of polyaniline doped with ferrocene perchlorate", Journal of Applied Polymer Science, vol. 102, pp. 5633-5639, 2006.

[7] E. Akbarinezhad, M. Ebrahimia, F. Sharif, M.M. Attar and H. R. Faridi, "Synthesis and evaluating corrosion protection effects of emeraldine base Pani/clay nanocomposite as a barrier pigment in zinc-rich ethyl silicate primer", Progress in Organic Coatings, vol. 70, pp. 39-44, 2011.

[8] A.B. Samui, A.S. Patankar, J. Rangarajan and P.C. Deb, "Study of polyaniline containing paint for corrosion prevention", Progress in Organic Coatings, vol. 47, pp. $1-7,2003$.

[9] M. Ates, "A review study of (bio) sensor systems based on conducting polymers", Materials Science and Engineering C, vol. 33, pp. 1853-1859, 2013.

[10] A. Habibi-Yangjeh, "Prediction dielectric constant of different ternary liquid mixtures at various temperatures and compositions using artificial neural networks", 
Physics and Chemistry of Liquids, vol.45, pp. 471-478, 2007.

[11] H. Kurt and M. Kayfeci, "Prediction of thermal conductivity of ethylene glycol-water solutions by using artificial neural networks", Applied Energy, vol. 86, pp. 2244-2248, 2009.

[12] K. Levenberg, "A Method for the Solution of Certain Non-Linear Problems in Least Squares" Quarterly of Applied Mathematics, vol. 2, pp. 164-168, 1944.

[13] D. Marquardt, “An Algorithm for Least-Squares Estimation of Nonlinear Parameters", SIAM Journal on Applied Mathematics, vol. 11, pp. 431-441, 1963.
[14] A.S. Nowick, A.V. Vaysleyb and I. Kuskovsky, "Universal dielectric response of variously doped $\mathrm{CeO}_{2}$ ionically conducting ceramics", Phys Rev B, vol. 58, pp. 8398-8406, 1998.

[15] V.F. Lvovich, Impedance Spectroscopy Applications to Electrochemical and Dielectric Phenomena, New Jersey: Wiley, 2012, ch. 4.

[16] J. Suchanicz, "The low-frequency dielectric relaxation $\mathrm{Na}_{0.5} \mathrm{Bi}_{0.5} \mathrm{TiO}_{3}$ ceramics" Mater Sci Eng B-Adv, vol. 55, pp. 114-118, 1998. 